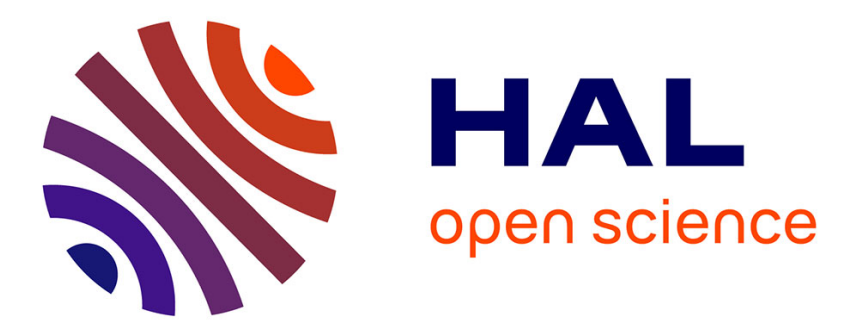

\title{
NEUMANN-DIRICHLET NASH STRATEGIES FOR THE SOLUTION OF ELLIPTIC CAUCHY PROBLEMS
}

Abderrahmane Habbal, Moez Kallel

\section{- To cite this version:}

Abderrahmane Habbal, Moez Kallel. NEUMANN-DIRICHLET NASH STRATEGIES FOR THE SOLUTION OF ELLIPTIC CAUCHY PROBLEMS. SIAM Journal on Control and Optimization, 2013, 51 (5), pp.4066-4083. hal-00923574

\section{HAL Id: hal-00923574 \\ https://hal.inria.fr/hal-00923574}

Submitted on 3 Jan 2014

HAL is a multi-disciplinary open access archive for the deposit and dissemination of scientific research documents, whether they are published or not. The documents may come from teaching and research institutions in France or abroad, or from public or private research centers.
L'archive ouverte pluridisciplinaire HAL, est destinée au dépôt et à la diffusion de documents scientifiques de niveau recherche, publiés ou non, émanant des établissements d'enseignement et de recherche français ou étrangers, des laboratoires publics ou privés. 


\title{
NEUMANN-DIRICHLET NASH STRATEGIES FOR THE SOLUTION OF ELLIPTIC CAUCHY PROBLEMS*
}

\author{
A. HABBAL ${ }^{\dagger}$ AND M. KALLEL $\ddagger$
}

\begin{abstract}
We consider the Cauchy problem for an elliptic operator, formulated as a Nash game. The overspecified Cauchy data are split between two players: the first player solves the elliptic equation with the Dirichlet part of the Cauchy data prescribed over the accessible boundary and a variable Neumann condition (which we call first player's strategy) prescribed over the inaccessible part of the boundary. The second player makes use correspondingly of the Neumann part of the Cauchy data, with a variable Dirichlet condition prescribed over the inaccessible part of the boundary. The first player then minimizes the gap related to the nonused Neumann part of the Cauchy data, and so does the second player with a corresponding Dirichlet gap. The two costs are coupled through a difference term. We prove that there always exists a unique Nash equilibrium, which turns out to be the reconstructed data when the Cauchy problem has a solution. We also prove that the completion Nash game has a stable solution with respect to noisy data. Some numerical two- and three-dimensional experiments are provided to illustrate the efficiency and stability of our algorithm.
\end{abstract}

Key words. elliptic Cauchy problem, Nash games, data completion

AMS subject classifications. 49J20, 65K10, 65N06, 90C30

DOI. $10.1137 / 120869808$

1. Introduction. We consider the following elliptic Cauchy problem:

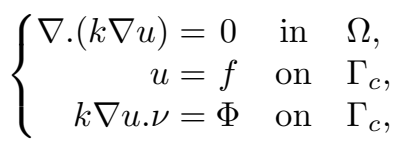

where $\Omega$ is a bounded open domain in $\mathbb{R}^{d}(d=2,3)$ with a sufficiently smooth boundary $\partial \Omega$ composed of two connected disjoint components $\Gamma_{c}$ and $\Gamma_{i}$. The parameters $k$, $f$, and $\Phi$ are given functions, and $\nu$ is the unit outward normal vector on the boundary. The Dirichlet data $f$ and the Neumann data $\Phi$ are the so-called Cauchy data, which are known on the accessible part $\Gamma_{c}$ of the boundary $\partial \Omega$, and the unknown field $u$ is the Cauchy solution.

The above Cauchy problem is also known as a data completion problem, where the data to be recovered, or missing data, are $u_{\mid \Gamma_{i}}$ and $k \nabla u . \nu_{\mid \Gamma_{i}}$, which are determined as soon as one knows $u$ in the whole domain $\Omega$. The Cauchy problem is a prototype of inverse boundary value problems (IBVP), which model a wide field of applications ranging from medical imaging to detection and nondestructive testing, and addressing quasi exhaustively all the fields of physics, from electromagnetism to acoustics and fluid and structural mechanics (see, e.g., $[9,10,13,17]$ ).

Classically, IBVP are known to be ill-posed. For instance, the solution of the Cauchy problem does not always exist for any pair of data $(f, \Phi)$, and if such a solution exists, it does not always depend continuously on the data (Hadamard's illposedness; see [21]). The Cauchy data $(f, \Phi)$ are called compatible (or consistent)

${ }^{*}$ Received by the editors March 12, 2012; accepted for publication (in revised form) August 6, 2013; published electronically October 28, 2013.

http://www.siam.org/journals/sicon/51-5/86980.html

${ }^{\dagger}$ Laboratoire J. A. Dieudonné, Université de Nice, and INRIA, Sophia Antipolis, France (habbal@ polytech.unice.fr).

${ }^{\ddagger}$ Laboratoire LAMSIN-ENIT, and IPEIT, Université de Tunis, Tunisia (moez.kallel@ipeit.rnu.tn). 
if the corresponding Cauchy problem (1.1) has a solution. (It is then unique thanks to classical continuation arguments.) Ill-posedness in the sense of Hadamard makes classical numerical methods usually inappropriate because they are unstable, and there is a need for carefully stabilized dedicated computational methods, sometimes by regularizing (through reformulation of) the Cauchy problem itself. Readers may refer to a wide literature dealing with the efficient numerical solution of elliptic Cauchy problems, e.g., $[4,14,15,16,18,23]$, and, dealing with the ill-posedness for the Cauchy problem, $[3,11,12]$ among many others.

Our purpose is to introduce an original method to solve the Cauchy problem, based on a game theory approach. We first recall in section 2 an optimal control formulation to solve the Cauchy problem used, e.g., in [1, 4, 23]. We then show in section 3 that the control formulation naturally leads to a Nash game of static nature with complete information, which involves Dirichlet gap and Neumann gap costs. The existence and uniqueness of the Nash equilibrium is proved, and when the Cauchy solution exists, it turns out that the Nash equilibrium is exactly the pair of missing data of the Cauchy problem; afterward, we end the section with a convergence result with respect to noisy data. Section 4 is devoted to sensitivity and implementation aspects, used to lead some numerical experiments. The numerical results are presented in section 5 to illustrate the efficiency of the present game-based approach. We end the paper with some concluding remarks.

The reader interested in some PDE-oriented applications of game theory may refer to $[19,20]$, where multidisciplinary topology optimization problems are formulated in words of Nash games; application to multiobjective shape optimization is considered in [26] and a multicriteria elliptic control problem where two independent controls play the role of strategies is studied in [25]. Refer to [8, 24, 28] for a general introduction and proof of convergence of computational methods for Nash equilibria, and refer to $[5,6,7]$ for a study of alternating algorithms which are closely linked to our present approach.

2. An optimal control formulation of the Cauchy problem. We assume that the boundary $\partial \Omega$ and the data $k, \Phi$, and $f$ are smooth enough, at least $\Omega$ has a piecewise $C^{1}$ boundary, and $(\Phi, f)$ belong to $H^{-\frac{1}{2}}\left(\Gamma_{c}\right) \times H^{\frac{1}{2}}\left(\Gamma_{c}\right)$. In this case, the Cauchy solution $u$, if it exists, belongs to the space $H^{1}(\Omega)$.

Many authors have formulated the Cauchy problem (1.1) as an optimal control one; see, e.g., $[1,4,23]$. The setting is as follows.

For given $\eta \in H^{-\frac{1}{2}}\left(\Gamma_{i}\right)$ and $\tau \in H^{\frac{1}{2}}\left(\Gamma_{i}\right)$, let us define $u_{1}(\eta)$ and $u_{2}(\tau)$ as the unique solutions in $H^{1}(\Omega)$ of the following elliptic boundary value problems:

$$
(\mathrm{SP} 1)\left\{\begin{array} { r l l } 
{ \nabla \cdot ( k \nabla u _ { 1 } ) = 0 } & { \text { in } \quad \Omega , } \\
{ u _ { 1 } = f } & { \text { on } \quad \Gamma _ { c } , } \\
{ k \nabla u _ { 1 } \cdot \nu = \eta } & { \text { on } \quad \Gamma _ { i } , }
\end{array} \quad ( \mathrm { SP } 2 ) \left\{\begin{array}{rl}
\nabla \cdot\left(k \nabla u_{2}\right)=0 & \text { in } \quad \Omega, \\
u_{2}=\tau & \text { on } \quad \Gamma_{i}, \\
k \nabla u_{2} \cdot \nu=\Phi & \text { on } \quad \Gamma_{c} .
\end{array}\right.\right.
$$

The optimization problem amounts to minimizing, among all Neumann-Dirichlet pairs $(\eta, \tau) \in H^{-\frac{1}{2}}\left(\Gamma_{i}\right) \times H^{\frac{1}{2}}\left(\Gamma_{i}\right)$, the following "Neumann-gap" cost:

$$
\mathcal{J}_{1}(\eta, \tau)=\mathrm{J}_{1}\left(\eta, \tau, u_{1}(\eta), u_{2}(\tau)\right)=\frac{1}{2}\left\|k \nabla u_{1} . \nu-\Phi\right\|_{H^{-\frac{1}{2}\left(\Gamma_{c}\right)}}^{2}+\frac{1}{2}\left\|u_{1}-u_{2}\right\|_{L^{2}\left(\Gamma_{i}\right)}^{2} .
$$

It can be easily proved that when the Cauchy problem (1.1) has a solution, then solving it is equivalent to solving the minimization problem

$$
\min _{(\eta, \tau) \in H^{-\frac{1}{2}}\left(\Gamma_{i}\right) \times H^{\frac{1}{2}}\left(\Gamma_{i}\right)} \mathcal{J}_{1}(\eta, \tau) .
$$


They also proved that the functional $\mathcal{J}_{1}$ is twice Fréchet differentiable and strictly convex.

The same conclusions above hold when a "Dirichlet-gap" cost is considered:

$$
\mathcal{J}_{2}(\eta, \tau)=\mathrm{J}_{2}\left(\eta, \tau, u_{1}(\eta), u_{2}(\tau)\right)=\frac{1}{2}\left\|u_{2}-f\right\|_{H^{\frac{1}{2}\left(\Gamma_{c}\right)}}^{2}+\frac{1}{2}\left\|u_{1}-u_{2}\right\|_{L^{2}\left(\Gamma_{i}\right)}^{2} .
$$

Let us remark that, depending on the authors, many other coupling terms were considered, among which are the difference of the normal derivatives of the fields over $\Gamma_{i}$ and distributed difference in the $L^{2}(\Omega)$ norm.

3. A Nash game formulation of the Cauchy problem. From the previous section, we remark that, formulated in the game theory language, the Neumann and Dirichlet controls $\eta$ and $\tau$ do cooperate to minimize either the Neumann-gap or the Dirichlet-gap costs. These two controls could as well cooperatively minimize any convex combination of the two costs $\mathcal{J}_{1}$ and $\mathcal{J}_{2}$.

Now, the fields $u_{1}(\eta)$ and $u_{2}(\tau)$ are aiming at the fulfillment of a possibly antagonistic goals, namely, minimizing the Neumann gap $\left\|k \nabla u_{1} \cdot \nu-\Phi\right\|_{H^{-\frac{1}{2}}\left(\Gamma_{c}\right)}$ and the Dirichlet gap $\left\|u_{2}-f\right\|_{H^{\frac{1}{2}\left(\Gamma_{c}\right)}}$. This antagonism is intimately related to Hadamard's ill-posedness character of the Cauchy problem and rises as soon as one requires that $u_{1}$ and $u_{2}$ coincide, which is exactly what the coupling term $\left\|u_{1}-u_{2}\right\|_{L^{2}\left(\Gamma_{i}\right)}$ is for. Thus, one may think of an iterative process which minimizes in a smart fashion the three terms, namely, Neumann-Dirichlet coupling terms.

Let us define the following two costs: for any $\eta \in H^{-\frac{1}{2}}\left(\Gamma_{i}\right)$ and $\tau \in H^{\frac{1}{2}}\left(\Gamma_{i}\right)$,

$$
\begin{aligned}
& J_{1}(\eta, \tau)=\frac{1}{2}\left\|k \nabla u_{1} \cdot \nu-\Phi\right\|_{H^{-\frac{1}{2}}\left(\Gamma_{c}\right)}^{2}+\frac{\alpha}{2}\left\|k \nabla u_{1} \cdot \nu-k \nabla u_{2} \cdot \nu\right\|_{H^{-\frac{1}{2}}\left(\Gamma_{i}\right)}^{2}, \\
& J_{2}(\eta, \tau)=\frac{1}{2}\left\|u_{2}-f\right\|_{H^{\frac{1}{2}}\left(\Gamma_{c}\right)}^{2}+\frac{\alpha}{2}\left\|k \nabla u_{1} \cdot \nu-k \nabla u_{2} \cdot \nu\right\|_{H^{-\frac{1}{2}}\left(\Gamma_{i}\right)}^{2},
\end{aligned}
$$

where the fields $u_{1}(\eta)$ and $u_{2}(\tau)$ are the unique solutions to (SP1) and (SP2), respectively, and $\alpha$ is a given positive parameter (e.g., $\alpha=1$ ). Differently from the definition of $\mathcal{J}_{1}, \mathcal{J}_{2}$ above, the coupling term is now the difference between the normal derivatives, but all our results do hold when considering the coupling term $\left\|u_{1}-u_{2}\right\|_{H^{\frac{1}{2}}\left(\Gamma_{i}\right)}$, for which the same proof techniques apply.

One may consider a decomposition-like method where the variable $\eta$ is used to minimize the Neumann gap + coupling term, in other words $J_{1}$, and $\tau$ is used to minimize the Dirichlet gap + coupling term, which defines $J_{2}$. Such a method fits into the area of mathematical games.

We shall say that there are two players, referred to as player 1 or Neumann gap, and player 2 or Dirichlet gap. Player 1 controls the strategy variable $\eta$ which belongs to the first player's strategy space $H^{-\frac{1}{2}}\left(\Gamma_{i}\right)$, and player 2 controls the strategy variable $\tau$ which belongs to the second player's strategy space $H^{\frac{1}{2}}\left(\Gamma_{i}\right)$. Each of the two players tries to minimize its own cost, namely, $J_{1}$ (Neumann gap + coupling term) for player 1 and $J_{2}$ (Dirichlet gap + coupling term) for player 2. As is classical, the fact that each player controls only his own strategy, while there is a strong dependance of each player's cost on the joint strategies $(\eta, \tau)$, justifies the use of the game theory framework (and terminology), a natural setting which may be used to formulate the negotiation between these two costs.

In order to be consistent with the classical formulation of the Cauchy problem, the relevant game theoretic framework to deal with is a static with complete information 
one, particularly because available Dirichlet and Neumann data are not considered in a leader-follower scheme. In this case, a commonly used solution concept (roughly speaking, in the game vocabulary, a rational and stable one) is the one of Nash equilibria, defined as follows.

Definition 3.1. A pair $\left(\eta_{N}, \tau_{N}\right) \in H^{-\frac{1}{2}}\left(\Gamma_{i}\right) \times H^{\frac{1}{2}}\left(\Gamma_{i}\right)$ is a Nash equilibrium for the two-player game involving the costs $J_{1}$ and $J_{2}$ if

$$
\begin{cases}J_{1}\left(\eta_{N}, \tau_{N}\right) \leq J_{1}\left(\eta, \tau_{N}\right) & \forall \eta \in H^{-\frac{1}{2}}\left(\Gamma_{i}\right), \\ J_{2}\left(\eta_{N}, \tau_{N}\right) \leq J_{2}\left(\eta_{N}, \tau\right) & \forall \tau \in H^{\frac{1}{2}}\left(\Gamma_{i}\right) .\end{cases}
$$

It is important to notice that the present game has a separable structure. Indeed, the players criteria are formed of individual costs, the Neumann gap depending only on $\eta$ for player 1 and the Dirichlet gap depending only on $\tau$ for player 2, plus a common coupling cost which depends on both $\eta$ and $\tau$. The game separable structure is crucial in our study, and we shall exploit it to prove that there exists a unique Nash equilibrium, which is shown to be the missing data when a Cauchy solution does exist. Based upon this structure of the criteria, we shall also establish a convergence result with respect to noisy data.

Let us start with the following preliminary proposition.

Proposition 3.2. The partial mapping $\eta \rightarrow J_{1}(\eta, \tau)$ (resp., $\tau \rightarrow J_{2}(\eta, \tau)$ ) is a quadratic strongly convex functional over $H^{-\frac{1}{2}}\left(\Gamma_{i}\right)$ (resp., $H^{\frac{1}{2}}\left(\Gamma_{i}\right)$ ).

Proof. Note that the field $u_{1}(\eta)$ is affine with respect to $\eta$, and so is the field $u_{2}(\tau)$ w.r.t. the variable $\tau$. Thus, the functions $J_{1}$ and $J_{2}$ are quadratic. Following, e.g., [1], it is an easy exercise to compute their second order differentials.

Let us consider the case of $J_{2}$, the case of $J_{1}$ being straightforward. First, notice that we can set

$$
u_{2}(\psi)=u_{2,0}(\psi)+u_{2, \Phi},
$$

where $u_{2,0}(\psi)$ solves the boundary value problem:

$$
\left\{\begin{aligned}
\nabla \cdot\left(k \nabla u_{2,0}\right)=0 & \text { in } \quad \Omega, \\
u_{2,0}=\psi & \text { on } \quad \Gamma_{i}, \\
k \nabla u_{2,0} . \nu=0 & \text { on } \quad \Gamma_{c} .
\end{aligned}\right.
$$

The second order differential of $J_{2}$ w.r.t. $\tau$ in any direction $\psi \in H^{\frac{1}{2}}\left(\Gamma_{i}\right)$ reads

$$
\left(d^{2} J_{2}(\eta, \tau) \cdot \psi, \psi\right)=\left\|u_{2,0}(\psi)\right\|_{H^{\frac{1}{2}}\left(\Gamma_{c}\right)}^{2}+\left\|k \nabla u_{2,0}(\psi) \cdot \nu\right\|_{H^{-\frac{1}{2}}\left(\Gamma_{i}\right)}^{2} \cdot
$$

It is immediate that if $\left(d^{2} J_{2}(\eta, \tau) \cdot \psi, \psi\right)=0$, then $u_{2,0}(\psi)=0$ over $\Gamma_{c}$, hence $u_{2,0}(\psi)$ vanishes over the whole $\Omega$ thanks to (3.4), so that $\psi=0$.

Indeed, strict convexity of $J_{1}$ and $J_{2}$ holds w.r.t. the pair $(\eta, \tau)$ as well. On the contrary, we shall see that only partial ellipticity (or coerciveness) of the costs holds, while it does not hold w.r.t. the pair $(\eta, \tau)$, precisely because of the coupling term.

Let us again focus on the case of $J_{2}$. (Uniform ellipticity of $J_{1}$ with respect to $\eta$ is explicit in its definition.)

Consider the following Steklov-Poincaré operator:

$$
\Lambda: H^{\frac{1}{2}}\left(\Gamma_{i}\right) \rightarrow H^{-\frac{1}{2}}\left(\Gamma_{i}\right): \quad u_{2,0}(\psi)_{\mid \Gamma_{i}}(=\psi) \mapsto\left(k \nabla u_{2,0}(\psi) . \nu\right)_{\mid \Gamma_{i}},
$$

where $u_{2,0}(\psi)$ solves the boundary value problem (3.4). 
The operator $\Lambda$ is a topological isomorphism (see [27]); thus its inverse $\Lambda^{-1}$ is continuous and there exists a constant $c>0$ such that for any $\psi$

$$
c\|\psi\|_{H^{\frac{1}{2}}\left(\Gamma_{i}\right)}=c\left\|\Lambda^{-1}\left(k \nabla u_{2,0}(\psi) \cdot \nu\right)\right\|_{H^{\frac{1}{2}}\left(\Gamma_{i}\right)} \leq\left\|k \nabla u_{2,0}(\psi) \cdot \nu\right\|_{H^{-\frac{1}{2}}\left(\Gamma_{i}\right)},
$$

which immediately yields the uniform strong convexity (uniform ellipticity) of the functional $J_{2}$ with respect to $\tau$.

It is important to notice that the partial ellipticity property of $\eta \rightarrow J_{1}(\eta, \tau)$ holds uniformly w.r.t. $\tau$, and conversely for $J_{2}$. It allows us to restrict the search for Nash equilibria to bounded subsets of the strategy spaces if necessary.

Now, following [6], let us introduce the functional $L(\eta, \tau)$ as follows:

$L(\eta, \tau)=\frac{1}{2}\left\|k \nabla u_{1} \cdot \nu-\Phi\right\|_{H^{-\frac{1}{2}}\left(\Gamma_{c}\right)}^{2}+\frac{1}{2}\left\|u_{2}-f\right\|_{H^{\frac{1}{2}\left(\Gamma_{c}\right)}}^{2}+\frac{\alpha}{2}\left\|k \nabla u_{1} \cdot \nu-k \nabla u_{2} \cdot \nu\right\|_{H^{-\frac{1}{2}}\left(\Gamma_{i}\right)}^{2}$.

The following theorem states that the Nash game is well defined and has a relevant interpretation in view of the Cauchy problem

TheOrem 3.3. Consider the Nash game defined by (3.1)-(3.2)-(3.3).

(i) There always exists a unique Nash equilibrium $\left(\eta_{N}, \tau_{N}\right) \in H^{-\frac{1}{2}}\left(\Gamma_{i}\right) \times H^{\frac{1}{2}}\left(\Gamma_{i}\right)$. It is also the minimum of $L(\eta, \tau)$ given by (3.5).

(ii) When the Cauchy problem has a solution $u$, then $u_{1}\left(\eta_{N}\right)=u_{2}\left(\tau_{N}\right)=u$, and $\left(\eta_{N}, \tau_{N}\right)$ are the missing data, namely, $\eta_{N}=k \nabla u . \nu_{\mid \Gamma_{i}}$ and $\tau_{N}=u_{\mid \Gamma_{i}}$.

Proof. The functional $L$ is made of parts of the costs $J_{1}$ and $J_{2}$. It is easy to check that it is strictly convex with respect to the pair $(\eta, \tau)$.

From the other part, any minimum of $L$ is a Nash equilibrium (for the costs $J_{1}$ and $J_{2}$ ) and conversely, thanks to the separable structure of the present game (consider the necessary optimality conditions).

We conclude that if a Nash equilibrium exists, then, as a minimum of the strictly convex $L$, it is unique.

The existence of a Nash equilibrium $\left(\eta_{N}, \tau_{N}\right) \in H^{-\frac{1}{2}}\left(\Gamma_{i}\right) \times H^{\frac{1}{2}}\left(\Gamma_{i}\right)$, id est a pair which fulfills (3.3), is obtained by a direct application of the Nash theorem: since strategy variables belong to Hilbert spaces, the uniform partial ellipticity of the costs allows for the choice of large enough closed bounded balls, which are then weakly compact convex sets, then continuity of the convex costs yields the weak lower semicontinuity over the so-defined balls.

Finally, note that if a Cauchy solution $u$ does exist, then by setting $\eta_{C}=k \nabla u \cdot \nu_{\mid \Gamma_{i}}$ and $\tau_{C}=u_{\mid \Gamma_{i}}$, one has immediately $L\left(\eta_{C}, \tau_{C}\right)=0$, since thanks to the uniqueness of the Cauchy solution, $u_{1}\left(\eta_{C}\right)=u_{2}\left(\tau_{C}\right)=u$. In this case, one has that $\left(\eta_{C}, \tau_{C}\right)$ is the minimum of the nonnegative functional $L$, and so it is also the Nash equilibrium $\left(\eta_{N}, \tau_{N}\right)$.

Let us now consider the case of noisy data. An important issue to consider when performing data completion is the stability (in the sense of robustness) of the identified solution with respect to the noise magnitude.

We assume that there exists a pair of compatible data $(f, \Phi)$ and denote by $u$ the corresponding Cauchy solution. We consider a family of not necessarily compatible data $\left(f^{\delta}, \Phi^{\delta}\right) \in H^{\frac{1}{2}}\left(\Gamma_{c}\right) \times H^{-\frac{1}{2}}\left(\Gamma_{c}\right)$ such that

$$
\left\|f^{\delta}-f\right\|_{H^{\frac{1}{2}\left(\Gamma_{c}\right)}}^{2}+\left\|\Phi^{\delta}-\Phi\right\|_{H^{-\frac{1}{2}}\left(\Gamma_{c}\right)}^{2} \leq \delta^{2} .
$$

For given $(\eta, \tau) \in H^{-\frac{1}{2}}\left(\Gamma_{i}\right) \times H^{\frac{1}{2}}\left(\Gamma_{i}\right)$, the fields $u_{1}^{\delta}(\eta)$ and $u_{2}^{\delta}(\tau)$ are the solution of the respective problems: 


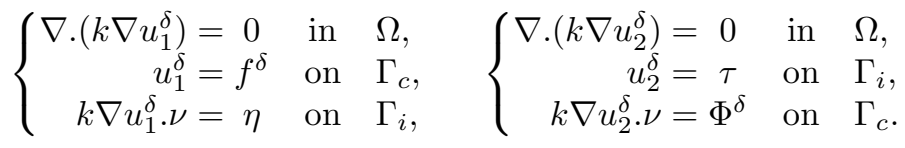

We define the associated cost functionals:

$$
\begin{aligned}
& J_{1}^{\delta}(\eta, \tau)=\frac{1}{2}\left\|k \nabla u_{1}^{\delta} \cdot \nu-\Phi^{\delta}\right\|_{H^{-\frac{1}{2}}\left(\Gamma_{c}\right)}^{2}+\frac{\alpha}{2}\left\|k \nabla u_{1}^{\delta} \cdot \nu-k \nabla u_{2}^{\delta} \cdot \nu\right\|_{H^{-\frac{1}{2}}\left(\Gamma_{i}\right)}^{2}, \\
& J_{2}^{\delta}(\eta, \tau)=\frac{1}{2}\left\|u_{2}^{\delta}-f^{\delta}\right\|_{H^{\frac{1}{2}}\left(\Gamma_{c}\right)}^{2}+\frac{\alpha}{2}\left\|k \nabla u_{1}^{\delta} \cdot \nu-k \nabla u_{2}^{\delta} \cdot \nu\right\|_{H^{-\frac{1}{2}}\left(\Gamma_{i}\right)}^{2} .
\end{aligned}
$$

The functions $J_{1}^{\delta}$ and $J_{2}^{\delta}$ enjoy the same properties than $J_{1}$ and $J_{2}$, claimed in Proposition 3.2, so there exists a unique corresponding Nash equilibrium $\left(\eta_{N}^{\delta}, \tau_{N}^{\delta}\right) \in$ $H^{-\frac{1}{2}}\left(\Gamma_{i}\right) \times H^{\frac{1}{2}}\left(\Gamma_{i}\right)$.

One may ask if, when $\delta \rightarrow 0$, the Nash equilibrium $\left(\eta_{N}^{\delta}, \tau_{N}^{\delta}\right)$ does converge to the missing data $\left(k \nabla u \cdot \nu_{\mid \Gamma_{i}}, u_{\mid \Gamma_{i}}\right)$ or, in other words, do the fields $u_{1}^{\delta}\left(\eta_{N}^{\delta}\right)$ and $u_{2}^{\delta}\left(\tau_{N}^{\delta}\right)$ converge to the Cauchy solution $u$ ?

The answer, positive, is given by the following theorem, which states that we have strong convergence of both the Nash equilibrium and fields sequences above.

THEOREM 3.4. Assume there exists a unique Cauchy solution $u \in H^{1}(\Omega)$ for a given compatible pair of data $(f, \Phi) \in H^{\frac{1}{2}}\left(\Gamma_{c}\right) \times H^{-\frac{1}{2}}\left(\Gamma_{c}\right)$.

Let $\left(f^{\delta}, \Phi^{\delta}\right) \in H^{\frac{1}{2}}\left(\Gamma_{c}\right) \times H^{-\frac{1}{2}}\left(\Gamma_{c}\right)$ be any sequence of noisy data such that

$$
\left\|f^{\delta}-f\right\|_{H^{\frac{1}{2}}\left(\Gamma_{c}\right)}^{2}+\left\|\Phi^{\delta}-\Phi\right\|_{H^{-\frac{1}{2}}\left(\Gamma_{c}\right)}^{2} \leq \delta^{2} .
$$

Then, the Nash game corresponding to the costs $J_{1}^{\delta}$ and $J_{2}^{\delta}$ defined by (3.8)-(3.9) has a unique Nash equilibrium $\left(\eta_{N}^{\delta}, \tau_{N}^{\delta}\right) \in H^{-\frac{1}{2}}\left(\Gamma_{i}\right) \times H^{\frac{1}{2}}\left(\Gamma_{i}\right)$ which strongly converges, as $\delta \rightarrow 0$, to the Cauchy missing data $\left(k \nabla u . \nu_{\mid \Gamma_{i}}, u_{\mid \Gamma_{i}}\right)$. Moreover, the solutions to (3.7), respectively $u_{1}^{\delta}\left(\eta_{N}^{\delta}\right)$ and $u_{2}^{\delta}\left(\tau_{N}^{\delta}\right)$, strongly converge in $H^{1}(\Omega)$ to the Cauchy solution $u$.

Proof. Let us again use the notation $\eta_{C}=k \nabla u \cdot \nu_{\mid \Gamma_{i}}$ and $\tau_{C}=u_{\mid \Gamma_{i}}$ the Cauchy missing data, and introduce the auxiliary functions

$$
z_{1}^{\delta}=u_{1}^{\delta}\left(\eta_{C}\right)-u \text { and } z_{2}^{\delta}=u_{2}^{\delta}\left(\tau_{C}\right)-u .
$$

The functions $z_{1}^{\delta}$ and $z_{2}^{\delta}$ are such that

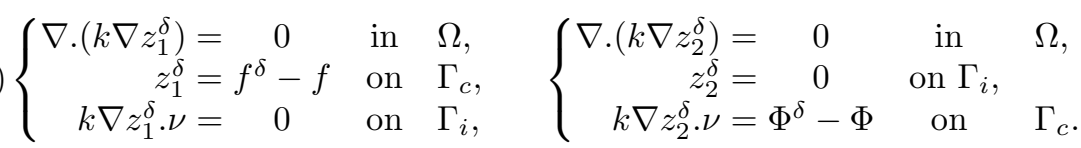

We introduce the corresponding perturbation of the $L$ functional given by (3.5):

$$
\begin{aligned}
L^{\delta}(\eta, \tau)= & \frac{1}{2}\left\|k \nabla u_{1}^{\delta}(\eta) \cdot \nu-\Phi^{\delta}\right\|_{H^{-\frac{1}{2}}\left(\Gamma_{c}\right)}^{2}+\frac{1}{2}\left\|u_{2}^{\delta}(\tau)-f^{\delta}\right\|_{H^{\frac{1}{2}}\left(\Gamma_{c}\right)}^{2} \\
& +\frac{\alpha}{2}\left\|k \nabla u_{1}^{\delta}(\eta) \cdot \nu-k \nabla u_{2}^{\delta}(\tau) \cdot \nu\right\|_{H^{-\frac{1}{2}}\left(\Gamma_{i}\right)}^{2} .
\end{aligned}
$$

Replacing $u_{1}^{\delta}\left(\eta_{C}\right)$ in (3.11) by $\left(z_{1}^{\delta}+u\right)$ and idem with $u_{2}^{\delta}\left(\tau_{C}\right)$, we obtain

$$
\begin{aligned}
L^{\delta}\left(\eta_{C}, \tau_{C}\right) \leq & \frac{1}{2}\left\|k \nabla\left(z_{1}^{\delta}-z_{2}^{\delta}\right) \cdot \nu\right\|_{H^{-\frac{1}{2}}\left(\Gamma_{c}\right)}^{2}+\frac{1}{2}\left\|z_{1}^{\delta}-z_{2}^{\delta}\right\|_{H^{\frac{1}{2}}\left(\Gamma_{c}\right)}^{2} \\
& +\frac{\alpha}{2}\left\|k \nabla\left(z_{1}^{\delta}-z_{2}^{\delta}\right) \cdot \nu\right\|_{H^{-\frac{1}{2}}\left(\Gamma_{i}\right)}^{2} \cdot
\end{aligned}
$$

Copyright (c) by SIAM. Unauthorized reproduction of this article is prohibited. 
Then, using the continuity of the trace operator from $H^{1}(\Omega)$ onto $H^{\frac{1}{2}}(\partial \Omega)$, and the continuity of the normal trace operator from $H^{1}(\Omega)$ onto $H^{-\frac{1}{2}}(\partial \Omega)$, it is easy to show that

$$
\begin{aligned}
L^{\delta}\left(\eta_{C}, \tau_{C}\right) & \leq\left(1+\frac{\alpha}{2}\right)\left\|z_{1}^{\delta}-z_{2}^{\delta}\right\|_{H^{1}(\Omega)}^{2} \\
& \leq(2+\alpha)\left(\left\|z_{1}^{\delta}\right\|_{H^{1}(\Omega)}^{2}+\left\|z_{2}^{\delta}\right\|_{H^{1}(\Omega)}^{2}\right) .
\end{aligned}
$$

Using a priori estimates on $z_{1}^{\delta}$ and on $z_{2}^{\delta}$, since $z_{1}^{\delta}$ fulfills the elliptic equation with nonhomogeneous Dirichlet condition $f^{\delta}-f$, and $z_{2}^{\delta}$ one with nonhomogeneous Neumann condition $\Phi^{\delta}-\Phi$, one gets

$$
L^{\delta}\left(\eta_{C}, \tau_{C}\right) \leq(2+\alpha)\left(\left\|f^{\delta}-f\right\|_{H^{\frac{1}{2}}\left(\Gamma_{c}\right)}^{2}+\left\|\Phi^{\delta}-\Phi\right\|_{H^{-\frac{1}{2}}\left(\Gamma_{c}\right)}^{2}\right) \leq(2+\alpha) \delta^{2} .
$$

We have previously seen that the Nash equilibrium $\left(\eta_{N}^{\delta}, \tau_{N}^{\delta}\right)$ is also the unique minimum of $L^{\delta}$; thus we have

$$
J_{1}^{\delta}\left(\eta_{N}^{\delta}, \tau_{N}^{\delta}\right) \leq L^{\delta}\left(\eta_{N}^{\delta}, \tau_{N}^{\delta}\right) \leq L^{\delta}\left(\eta_{C}, \tau_{C}\right) \leq(2+\alpha) \delta^{2}
$$

and $J_{2}^{\delta}\left(\eta_{N}^{\delta}, \tau_{N}^{\delta}\right) \leq(2+\alpha) \delta^{2}$ as well.

Now, since the mapping $\eta \rightarrow J_{1}^{\delta}(\eta, \tau)$ is coercive, uniformly in $\tau$ (and in $\delta$ ), and since the same corresponding property holds for $\tau \rightarrow J_{2}^{\delta}(\eta, \tau)$, we obtain that the sequence $\left(\eta_{N}^{\delta}\right)$ is uniformly bounded in $H^{-\frac{1}{2}}\left(\Gamma_{i}\right)$ and $\left(\tau_{N}^{\delta}\right)$ is uniformly bounded in $H^{\frac{1}{2}}\left(\Gamma_{i}\right)$. Thus, up to a subsequence, we have from one part that $\eta_{N}^{\delta}$ converges weakly to some $\eta_{N}^{0} \in H^{-\frac{1}{2}}\left(\Gamma_{i}\right)$ and from other part, the sequence $\tau_{N}^{\delta}$ converges weakly to some $\tau_{N}^{0} \in H^{\frac{1}{2}}\left(\Gamma_{i}\right)$.

Since by assumption (3.6), as $\delta$ goes to zero, the sequence $f^{\delta}$ strongly converges to $f$ in $H^{\frac{1}{2}}\left(\Gamma_{c}\right)$ and $\Phi^{\delta}$ strongly converges to $\Phi$ in $H^{-\frac{1}{2}}\left(\Gamma_{c}\right)$, we conclude that the sequences $u_{1}^{\delta}\left(\eta_{N}^{\delta}\right)$ and $u_{2}^{\delta}\left(\tau_{N}^{\delta}\right)$, which are the solutions to (3.7), weakly converge in $H^{1}(\Omega)$ to respectively $\bar{u}_{1}\left(\eta_{N}^{0}\right)$ and $\bar{u}_{2}\left(\tau_{N}^{0}\right)$, which are the unique solutions to the respective equations

$$
\left\{\begin{array} { r l l } 
{ \nabla \cdot ( k \nabla \overline { u } _ { 1 } ) = 0 } & { \text { in } } & { \Omega , } \\
{ \overline { u } _ { 1 } = f } & { \text { on } } & { \Gamma _ { c } , } \\
{ k \nabla \overline { u } _ { 1 } \cdot \nu = \eta _ { N } ^ { 0 } } & { \text { on } } & { \Gamma _ { i } , }
\end{array} \quad \left\{\begin{array}{rlll}
\nabla \cdot\left(k \nabla \bar{u}_{2}\right)=0 & \text { in } & \Omega, \\
\bar{u}_{2}=\tau_{N}^{0} & \text { on } & \Gamma_{i}, \\
k \nabla \bar{u}_{2} \cdot \nu=\Phi & \text { on } & \Gamma_{c} .
\end{array}\right.\right.
$$

Taking $\delta \rightarrow 0$ in (3.16) yields that $\left(k \nabla u_{1}^{\delta} . \nu-\Phi^{\delta}\right)$ strongly converges to 0 in $H^{-\frac{1}{2}}\left(\Gamma_{c}\right)$, which means that $\left(k \nabla u_{1}^{\delta} . \nu\right)$ strongly converges to $\Phi$.

Hence, $k \nabla \bar{u}_{1} . \nu=\Phi$ over $\Gamma_{c}$.

Thanks to the uniqueness of the Cauchy problem, we conclude that $\bar{u}_{1}=u$. The same reasoning applied to the first term in $J_{2}^{\delta}$ would directly yield $\bar{u}_{2}=f$ over $\Gamma_{c}$ and thanks to (3.17), we conclude that the fields $\bar{u}_{1}$ and $\bar{u}_{2}$ are equal to the Cauchy solution $u$.

Finally, observe that $L^{\delta}\left(\eta_{N}^{\delta}, \tau_{N}^{\delta}\right)$ tends to zero with $\delta$, which expresses that the trace of the difference $\left(u_{1}^{\delta}-u_{2}^{\delta}\right)$ strongly converges to zero in $H^{\frac{1}{2}}\left(\Gamma_{c}\right)$, and the trace of its normal derivative strongly converges to zero in $H^{-\frac{1}{2}}\left(\Gamma_{i}\right)$. Hence, $\left(u_{1}^{\delta}-u_{2}^{\delta}\right)$ strongly converges to zero in $H^{1}(\Omega)$ and then its trace strongly converges to zero over $H^{\frac{1}{2}}\left(\Gamma_{i}\right)$ as well.

Using (3.17) implies that the trace of $u_{1}^{\delta}$ strongly converges to $\tau_{N}^{0}$ in $H^{\frac{1}{2}}\left(\Gamma_{i}\right)$, and since $k \nabla \bar{u}_{1} . \nu=\Phi$ over $\Gamma_{c}$, it is then easy to check that $u_{1}^{\delta}$ indeed strongly converges to $\bar{u}_{2}=u$. The same conclusion holds for $u_{2}^{\delta}$. 
Consequently, as traces of strongly convergent sequences, we have then proved that the sequence $\left(\eta_{N}^{\delta}\right)$ strongly converges in $H^{-\frac{1}{2}}\left(\Gamma_{i}\right)$ to $\eta_{N}^{0}=\eta_{C}=k \nabla u \cdot \nu_{\mid \Gamma_{i}}$ and that the sequence $\left(\tau_{N}^{\delta}\right)$ strongly converges in $H^{\frac{1}{2}}\left(\Gamma_{i}\right)$ to $\tau_{N}^{0}=\tau_{C}=u_{\mid \Gamma_{i}}$.

4. Numerical procedure. From the computational viewpoint, in [6] the authors propose an alternating minimization algorithm, also referred to as the inertial Nash equilibration process, to compute the the Nash equilibrium by means of the following iterative process.

Let $\left(\eta^{0}, \tau^{0}\right)$ be a given initial state

$$
\left\{\begin{array}{l}
\eta^{(k+1)}=\operatorname{argmin}_{\eta}\left\{J_{1}\left(\eta, \tau^{(k)}\right)+\frac{\beta}{2} \int_{\Gamma_{i}}\left(\eta-\eta^{(k)}\right)^{2} d \Gamma_{i}\right\}, \\
\tau^{(k+1)}=\operatorname{argmin}_{\tau}\left\{J_{2}\left(\eta^{(k+1)}, \tau\right)+\frac{\beta}{2} \int_{\Gamma_{i}}\left(\tau-\tau^{(k)}\right)^{2} d \Gamma_{i}\right\},
\end{array}\right.
$$

where $\beta$ is a given positive parameter (e.g., $\beta=1$ ).

In [6], the convergence of the alternating algorithm above is proved under suitable assumptions which also hold in our case; see Proposition 3.2.

Our algorithm is written as follows:

Set $k=0$. Starting from an initial guess $S^{(0)}=\left(\eta^{(0)}, \tau^{(0)}\right)$ :

Step 1: Compute $\bar{\eta}^{(k)}$, which solves $\min _{\eta} J_{1}\left(\eta, \tau^{(k)}\right)$

$$
\text { and set } \eta^{(k+1)}=t \eta^{(k)}+(1-t) \bar{\eta}^{(k)}, 0<t<1 \text {. }
$$

Step 2: Compute $\bar{\tau}^{(k)}$, which solves $\min _{\tau} J_{2}\left(\eta^{(k+1)}, \tau\right)$ and set $\tau^{(k+1)}=t \tau^{(k)}+(1-t) \bar{\tau}^{(k)}, 0<t<1$.

Step 3: Set $S^{(k+1)}=\left(\eta^{(k+1)}, \tau^{(k+1)}\right)$.

Redo (Step 1) until the sequence $S^{(k)}$ converges.

As a stopping criterion we used the classical stationarity one,

$$
\left\|S^{(k+1)}-S^{(k)}\right\| \leq \epsilon,
$$

where $\epsilon$ is given small enough tolerance. The relaxation parameter $t$ above is used to accelerate the convergence of the alternating algorithm, whose convergence rate is relatively slow. (The expected convergence speed may be drawn from the proof of Propositon 4.4 in [5].) One may also consider parallel implementations for the computation of a Nash equilibrium in order to save overall computation time. Such distributed algorithms have been studied in, e.g., [8] and [24].

It is easy to show that the above procedure is equivalent to the algorithm (4.1) as soon as one uses a fixed step gradient method to solve the partial optimization problems in Step 1 and Step 2 above. For the numerical problem, we used the discretized version which was proved to converge in [28].

To this end, the gradients may be efficiently computed by means of an adjoint state method. Let us define, for the case of, e.g., $J_{1}$, the following Lagrangian:

$$
\begin{aligned}
\mathcal{L}\left(\eta, \tau, \tau^{*}, u_{1}, u_{2}, \lambda_{1}, \lambda_{2}\right)= & \frac{1}{2}\left\|\left(k \nabla u_{1} \cdot \nu-\Phi\right)\right\|_{H^{-\frac{1}{2}}\left(\Gamma_{c}\right)}^{2}+\frac{\alpha}{2}\left\|\left(\eta-k \nabla u_{2} \cdot \nu\right)\right\|_{H^{-\frac{1}{2}}\left(\Gamma_{i}\right)}^{2} \\
& +\int_{\Omega} k \nabla u_{1} \cdot \nabla \lambda_{1} d \Omega-\int_{\Gamma_{i}} \eta \lambda_{1} d \Gamma_{i} \\
& +\int_{\Omega} k \nabla u_{2} \cdot \nabla \lambda_{2} d \Omega-\int_{\Gamma_{c}} \Phi \lambda_{2} d \Gamma_{c} \\
& +\int_{\Gamma_{i}} \tau^{*}\left(u_{2}-\tau\right) d \Gamma_{i}
\end{aligned}
$$

Copyright ( ) by SIAM. Unauthorized reproduction of this article is prohibited. 
where $(\eta, \tau) \in H^{-\frac{1}{2}}\left(\Gamma_{i}\right) \times H^{\frac{1}{2}}\left(\Gamma_{i}\right),\left(u_{1}, u_{2}, \lambda_{1}, \lambda_{2}\right) \in H^{1}(\Omega) \times H^{1}(\Omega) \times W_{1} \times W_{2}$ and $\tau^{*} \in H^{-\frac{1}{2}}\left(\Gamma_{i}\right)$, where the latter two spaces are given by

$$
W_{1}=\left\{v \in H^{1}(\Omega) \text { such that }\left.v\right|_{\Gamma_{c}}=0\right\} \quad \text { and } \quad W_{2}=H^{1}(\Omega) .
$$

The Lagrangian is used to compute the gradients $\nabla_{\eta} J_{1}$ and $\nabla_{\tau} J_{2}$. (Wherever it is involved, the inner product in the Hilbert space $H^{-\frac{1}{2}}\left(\Gamma_{i}\right)$ is represented using a classical integral notation.)

Proposition 4.1. We have the following two partial derivatives:

$$
\left\{\begin{array}{l}
\frac{\partial J_{1}}{\partial \eta}(\eta, \tau) \xi=-\int_{\Gamma_{i}}\left(\lambda_{1}-\alpha\left(\eta-k \nabla u_{2} . \nu\right)\right) \xi d \Gamma_{i} \quad \forall \xi \in H^{-\frac{1}{2}}\left(\Gamma_{i}\right), \\
\text { where } \lambda_{1} \in W_{1} \text { solves the adjoint problem } \\
\int_{\Omega} k \nabla \lambda_{1} . \nabla \gamma d \Omega=-\int_{\Gamma_{c}}\left(k \nabla u_{1} . \nu-\Phi\right)(k \nabla \gamma \cdot \nu) d \Gamma_{c}, \quad \gamma \in W_{1},
\end{array}\right.
$$

and

$$
\left\{\begin{array}{l}
\frac{\partial J_{2}}{\partial \tau}(\eta, \tau) h=\int_{\Gamma_{i}}\left(k \nabla \lambda_{2} . \nu\right) h d \Gamma_{i}, \quad h \in H^{\frac{1}{2}}\left(\Gamma_{i}\right), \\
\text { where } \lambda_{2} \in W_{2} \text { solves the adjoint problem } \\
\begin{cases}\nabla \cdot k \nabla \lambda_{2}=0 & \text { in } \quad \Omega, \\
k \nabla \lambda_{2} \cdot \nu=f-u_{2} & \text { on } \Gamma_{c}, \\
\lambda_{2}=-\alpha\left(\eta-k \nabla u_{2} . \nu\right) & \text { on } \Gamma_{i} .\end{cases}
\end{array}\right.
$$

5. Numerical results. The computational methodology used to illustrate the efficiency of the present approach is classical. All experiments are performed on a personal computer and all the PDEs are numerically solved using FreeFem $++[22]$, a finite element based free software. In order to obtain accurate approximations of the normal derivatives of $u_{1}, u_{2}$, and of $\lambda_{2}$, the dual Raviart-Thomas mixed finite elements are used.

We consider a domain $\Omega$ defined as the open bounded set delimited by two concentric circles in two-dimensional (2D) test cases or two concentric spheres in 3D test cases. The inner boundary plays the role of $\Gamma_{i}$, where the trace and normal derivative are missing, and the outer one plays the one of $\Gamma_{c}$ where the latter information is over specified.

We then consider explicit well-known analytical solutions, generically denoted by $u$, which are harmonic inside the domain $\Omega$, and set the trace and normal derivative of $u$ over $\Gamma_{c}$ as being the measured data $f=u_{\mid \Gamma_{c}}$ and $\Phi=(k \nabla u . \nu)_{\mid \Gamma_{c}}$. We sometimes refer to these data as temperature and flux (with obvious interpretation).

In order to test the robustness of the proposed method we add white noise to the temperature $f$ and the heat flux $\Phi$ as follows:

$$
f^{\sigma}=u+\sigma w_{1} \quad \text { and } \quad \Phi^{\sigma}=k \nabla u . \nu+\sigma w_{2} \quad \text { on } \quad \Gamma_{c},
$$

where $\sigma$ denotes the noise level relative to $\|\cdot\|_{L^{2}\left(\Gamma_{c}\right)}$ of $u$ and $k \nabla u . \nu$, respectively, whereas $\left(w_{1}, w_{2}\right)$ are normelly distributed random functions.

Our algorithm performs a denoising task on the noisy prescribed Cauchy data $f^{\sigma}$ and $\Phi^{\sigma}$. For instance, let us denote by $\left(\tau_{N}^{\sigma}, \eta_{N}^{\sigma}\right)$ the Nash equilibrium associated to the latter noisy over specified data. Then, the pair of optimal solutions $\left(\left.u_{2}\left(\tau_{N}\right)\right|_{\Gamma_{c}},\left.\left(k \nabla \tilde{u}_{1} . \nu\right)\right|_{\Gamma_{c}}\right)$ may be viewed as regularized Cauchy data obtained from the noisy Cauchy data, where $\tilde{u_{1}}$ is the solution of 


$$
\left\{\begin{array}{lll}
\nabla \cdot k \nabla \tilde{u}_{1}=0 & \text { in } & \Omega, \\
\tilde{u}_{1}=u_{2}\left(\tau_{N}\right) & \text { on } & \Gamma_{c} \\
k \nabla \tilde{u}_{1} . \nu=\eta_{N} & \text { on } & \Gamma_{i} .
\end{array}\right.
$$

We present numerical results which illustrate the stability of our method with respect to noisy data, as well as an example of the noise deblurring property outlined above. The presented graphics are related to the profiles over $\Gamma_{i}$ of the Dirichlet and Neumann missing data and to the fields $u_{1}$ and $u_{2}$. We also provide convergence relative errors history, related to the fields and to the missing profiles, as a function of iterations and for different noise levels. We also present a stationarity history for the Nash overall computation iterations.

The computation of the Nash equilibrium is performed as described in section 4, where the partial optimization tasks of Step 1 and Step 2 use a fixed line-search gradient method. Here, during the early Nash steps, too accurate best response of each player to the other's strategy is not necessary; it is sufficient to carry out only a few iterations in the optimization process. The number of iterations is then increased as the process converges to the Nash equilibrium. A second formulation would be obtained if we assume that the players compute not sequentially, but in parallel with Step 1; in Step 2 we compute $\bar{\tau}^{(k)}$, which solves $\min _{\tau} J_{2}\left(\eta^{(k)}, \tau\right)$.

An arbitrary initial guess such as $S^{(0)}=\left(\eta^{(0)}, \tau^{(0)}\right)=(0,0)$ is chosen to start the algorithm, the physical function $k$ takes the constant value 1 in $\Omega$, and the parameters $\alpha$ (weight of the coupling term in the costs), $t$ (relaxation parameter in the computation of Nash equilibrium), and $\epsilon$ (stopping criteria) are set to $\alpha=1, t=0.25$ and $\epsilon=0.002$.

5.1. Two 2D test cases. Two test examples are selected from the literature, particularly from [1] and references therein.

We consider an annular domain $\Omega$ with circular boundary components $\Gamma_{i}$ and $\Gamma_{c}$, both centered at $(0,0)$ and with radii $R_{i}=0.6$ and $R_{c}=1$, respectively.

Test case A. The first 2D experiment is related to a smooth case. The artificial Cauchy data $f$ and $\Phi$ are defined as respectively the trace and the normal derivative, over the circle $\Gamma_{c}$, of the harmonic function:

$$
u(x, y)=e^{x} \cos (y) .
$$

In this example, the finite element discretization of the domain boundary is made of 180 outer vertices and 90 inner vertices.

In Figure 5.1, the missing data $\tau_{N}$ and $\eta_{N}$ are presented at convergence of the algorithm (4.1) dedicated to the computation of the Nash equilibrium for different noise levels $\sigma$. The obtained Dirichlet as well as Neumann profiles show remarkable stability with respect to noise.

The denoising effect, through computing the Nash equilibrium and solving (5.2), is actually observed in Figure 5.2 for a noise level of $5 \%$.

Test case B. The second 2D experiment is related to the singular function:

$$
u(x, y)=\operatorname{Re}\left(\frac{1}{z-a}\right), \quad \text { where } z=x+i y .
$$

In this case, the singularity source, located at $a=(0.5,0)$, is in the vicinity of the circle $\Gamma_{i}$, and reconstruction of the solution over this boundary is a numerically challenging task, particularly in the case of noisy data. Here refinement of the mesh is done by 

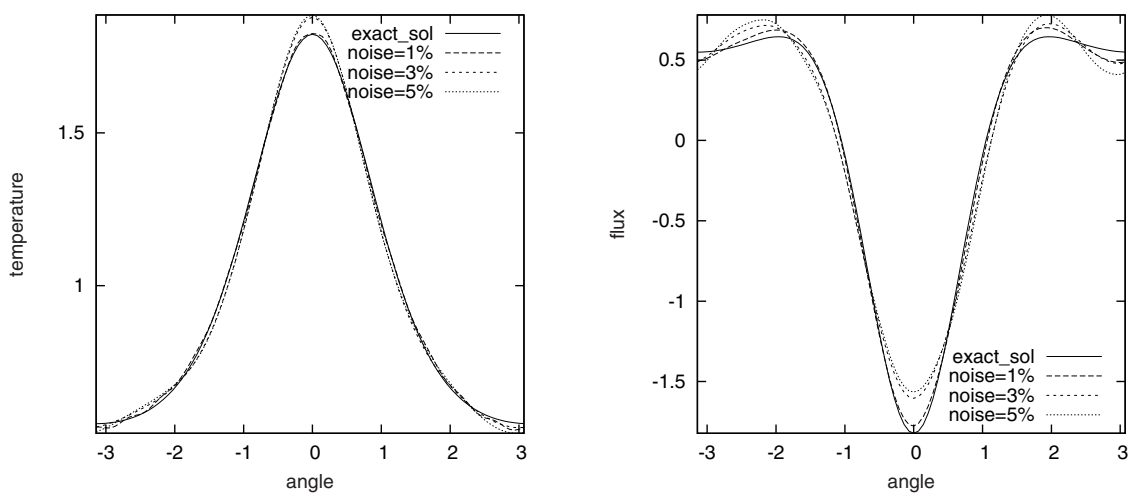

Fig. 5.1. Test case A. Reconstructed smooth Dirichlet $\left(\tau_{N}\right.$, left) and Neumann $\left(\eta_{N}\right.$, right) data over $\Gamma_{i}$. The profiles are presented at convergence and for various noise levels $\sigma \in\{1 \%, 3 \%, 5 \%\}$. The corresponding traces of the exact solution are also plotted. The finite element computations are performed with 1529 nodes and 2788 triangles.
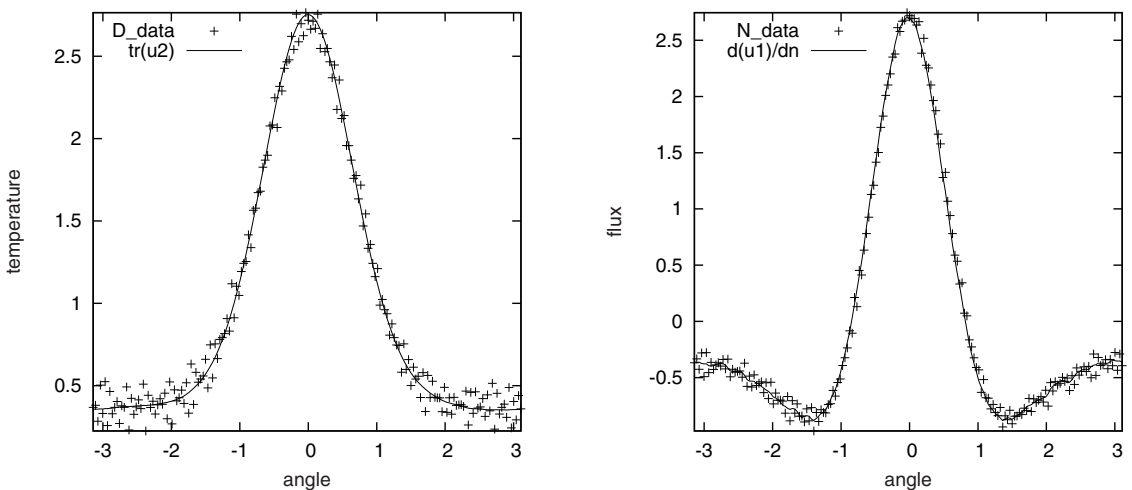

Fig. 5.2. Test case A. Regularization of noisy Cauchy data (noise level is $\sigma=5 \%$ ). At convergence: (left) the smoothed profile $\left.u_{2}\left(\tau_{N}\right)\right|_{\Gamma_{c}}$ (- line) is compared to the random $f^{\sigma}$ ( + dots); (right) the smoothed flux profile $\left.k \nabla \tilde{u_{1}} \cdot \nu\right|_{\Gamma_{c}}\left(-\right.$ line) is compared to $\Phi^{\sigma}$ ( + dots).

increasing the number of vertices on the boundary, we used 320 vertices on $\Gamma_{c}$ and 180 vertices on $\Gamma_{i}$.

The results in Figure 5.3 show again the stability of our method. The profile shape is well captured, including the localization of the singularity peak, whose magnitude, however, is underestimated for the trace as well as for the normal derivative.

Finally, we led a comparison of our algorithm with the control-type method introduced in [1]. This method was recently extended to the Stokes system in [2]. The proposed algorithm in [1] solves the classical minimization problem (2.3) with a stopping criterion

$$
\left\|u_{2}-f^{\sigma}\right\|_{L^{2}\left(\Gamma_{c}\right)} \leq \varepsilon
$$

where $\varepsilon$ is given tolerance error.

To compare the sensitivity to the noise level $\sigma$ defined in (5.1), using the controltype method [1] and our game method, numerical solutions were computed for test case A for different values of $\sigma$.

Copyright (C) by SIAM. Unauthorized reproduction of this article is prohibited. 

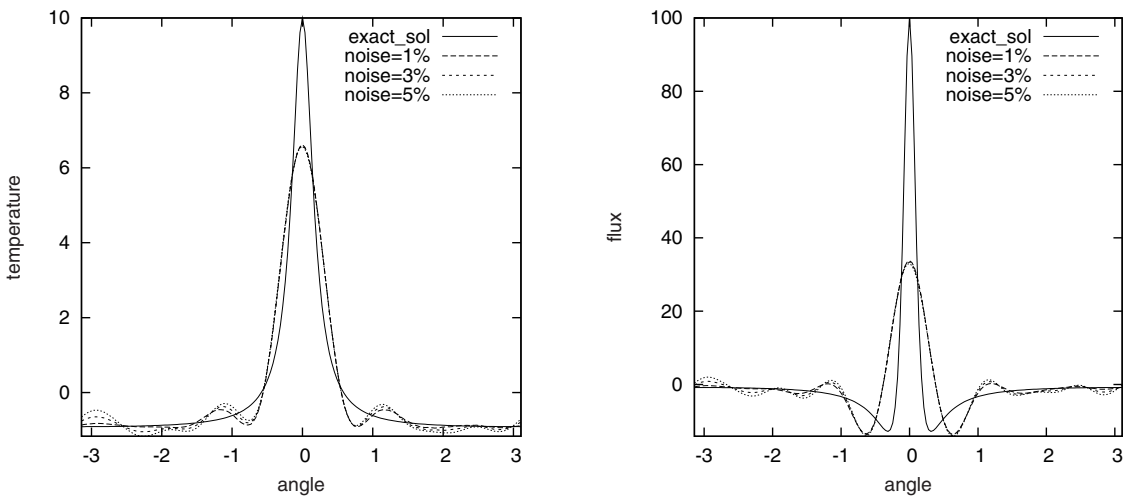

FIG. 5.3. Test case B. Reconstructed singular Dirichlet $\left(\tau_{N}\right.$, left) and Neumann $\left(\eta_{N}\right.$, right) data over $\Gamma_{i}$. The profiles are presented at convergence and for various noise levels $\sigma \in\{1 \%, 3 \%, 5 \%\}$. The corresponding traces of the exact solution are also plotted. The finite element computations are performed with 5405 nodes and 10,310 triangles.

TABLE 5.1

Test case A. Control type (classic) compared to Nash (game) algorithms. Relative $L^{2}$-errors on missing Dirichlet data $\left\|\tau_{N}-u_{\mid \Gamma_{i}}\right\| /\left\|u_{\mid \Gamma_{i}}\right\|$ and Neumann data $\left\|\eta_{N}-\frac{\partial u}{\partial \nu}\left|\Gamma_{i}\|/\| \frac{\partial u}{\partial \nu}\right|_{\Gamma_{i}}\right\|$ are shown for various noise levels.

\begin{tabular}{|c|c|c|c|}
\hline Noise level & $\sigma=2 \%$ & $\sigma=5 \%$ & $\sigma=8 \%$ \\
\hline Reconstructed data & Dirichlet-Neumann & Dirichlet-Neumann & Dirichlet-Neumann \\
\hline Classic method & $0.0222-0.1242$ & $0.0387-0.2242$ & $0.0573-0.3590$ \\
\hline Nash game method & $0.0196-0.1175$ & $0.0253-0.1619$ & $0.0338-0.2169$ \\
\hline
\end{tabular}

Table 5.1 represents the $L^{2}$-errors on the reconstructed missing data for $\sigma$ equal to, respectively, $2 \%, 5 \%$, and $8 \%$. For $\sigma=8 \%$ a remarkable advantage is observed for the game method in the reconstructed Neumann data. These differences are also observed in Figure 5.4.

5.2. Two 3D test cases. As for the $2 \mathrm{D}$ case, we consider a thick spherical shell domain $\Omega$ with boundary components $\Gamma_{i}$ and $\Gamma_{c}$, which are two spheres both centered at $(0,0,0)$ and with radii given by, respectively, $R_{i}=0.6$ and $R_{c}=1$.
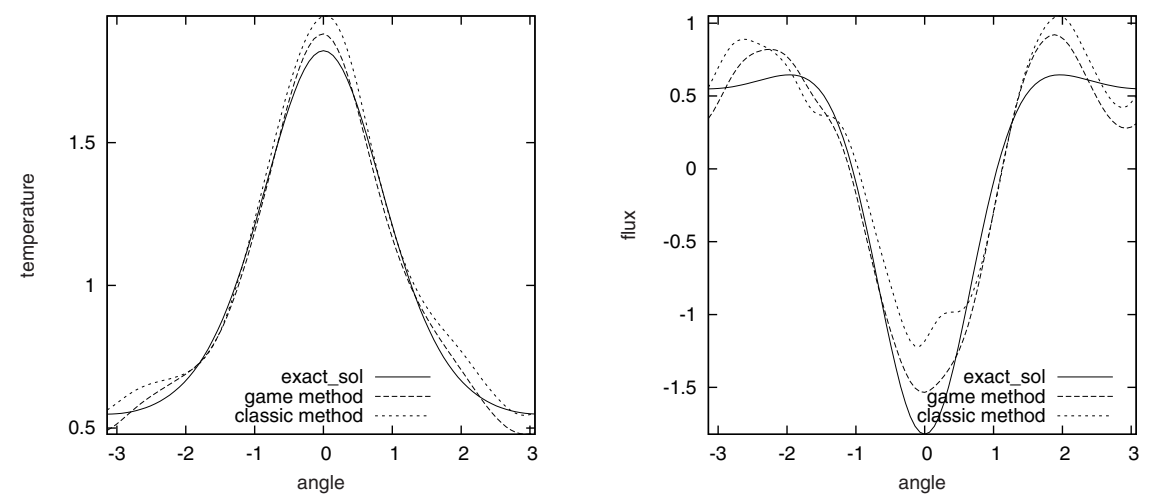

FIG. 5.4. Test case A. Control-type (classic) compared to Nash (game) algorithms for high noise level $\sigma=8 \%$. Reconstructed Dirichlet (left) and Neumann (right) data over $\Gamma_{i}$; the two are compared to the exact solution.

Copyright $@$ by SIAM. Unauthorized reproduction of this article is prohibited. 

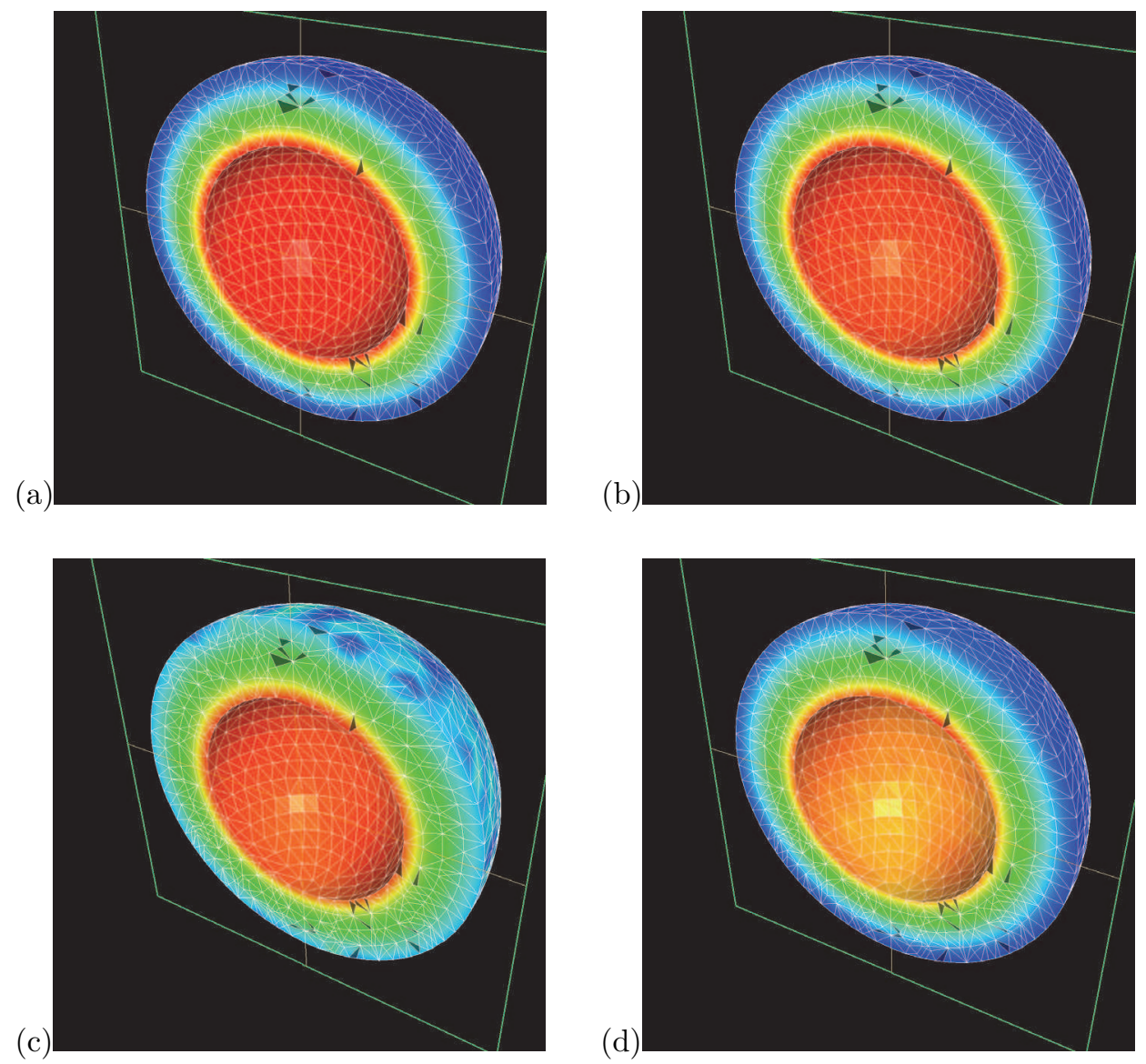

Fig. 5.5. Test case C. Top row: noise level is 0\%. At convergence, we plot a plane slice of the level sets of (a) $u_{1}$ and (b) $u_{2}$. Bottom row: noise level is 5\%. At convergence, we plot the level sets of (c) $u_{1}$ and (d) $u_{2}$. The finite element computations are performed with 4740 nodes and 22,795 tetrahedral elements.

Again two functions, denoted by $u$, are selected to play the role of exact solutions to the Cauchy problem for the Laplace operator. To this end, the Cauchy data $f$ and $\Phi$ are defined as, respectively, the trace and normal derivative of the involved functions over the sphere $\Gamma_{c}$.

Test case $\mathbf{C}$. The first function is radial, so it is of constant trace over each of the spherical components of the boundary:

$$
u(x, y, z)=\frac{1}{\sqrt{x^{2}+y^{2}+z^{2}}} .
$$

The function $u$ given by (5.4) is the solution of $\Delta u=\delta_{0}$, where $\delta_{0}$ is the Dirac distribution at the origin $(0,0,0)$, a point source that is not in $\Omega$, so $u$ is harmonic and smooth enough inside $\Omega$.

In Figure 5.5, level set slices are shown for the fields $u_{1}$ and $u_{2}$ at convergence for noise-free and 5\% noisy data. The overall Nash algorithm (4.1) converged in 150 iterations. More detailed issues related to the convergence are presented in Figure 5.6. Relative $L^{2}$-errors behave well in the noise-free and noisy cases. The relative errors on 

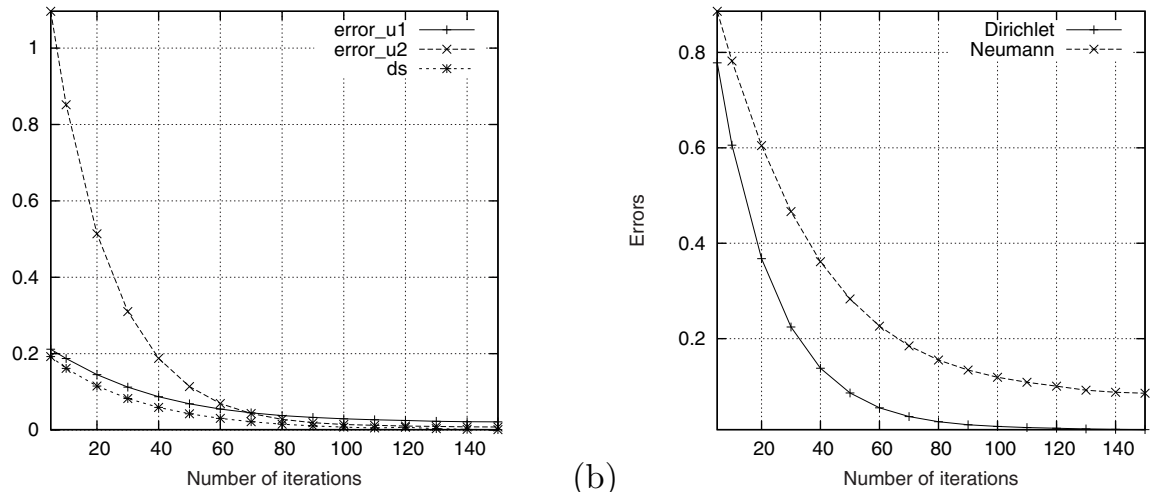

(a)

(b)
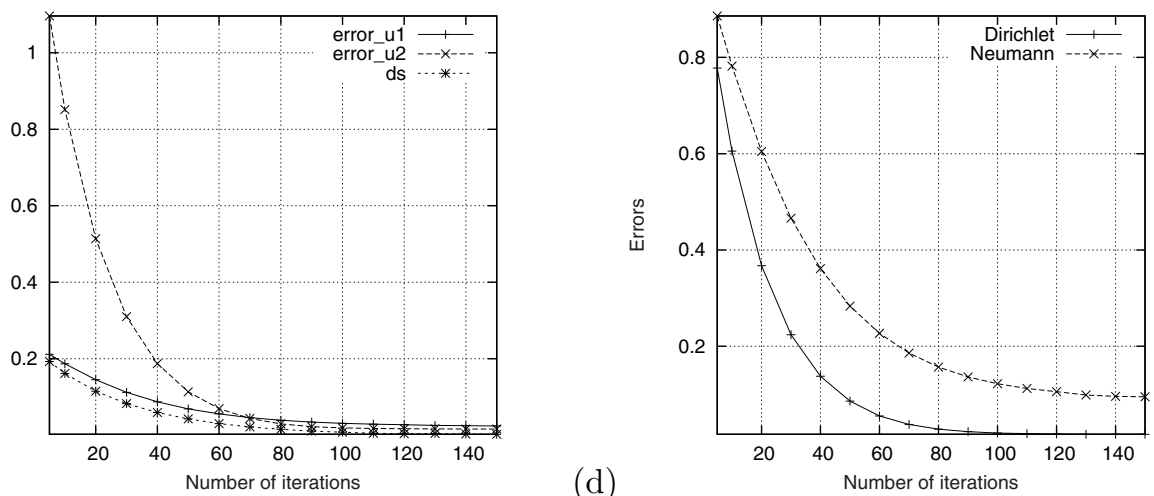

Fig. 5.6. Test case C. Top row: Relative $L^{2}$-errors are presented as a function of overall Nash iterations $k$ for a noise level $\sigma=0 \%$. (a) reconstructed fields: $\left\|u_{i}^{(k)}-u\right\| /\|u\|, i=1,2$, and Nash strategies $d s=\left\|S^{(k)}-S^{(k-1)}\right\|$; (b) missing Dirichlet data: $\left\|\tau^{(k)}-u_{\mid \Gamma_{i}}\right\| /\left\|u_{\mid \Gamma_{i}}\right\|$ and Neumann data $\left\|\eta^{(k)}-\frac{\partial u}{\partial \nu}{ }_{\mid \Gamma_{i}}\right\| /\left\|\left.\frac{\partial u}{\partial \nu}\right|_{\Gamma_{i}}\right\|$. Bottom row: the corresponding relative errors for a noise level $\sigma=5 \%$ are plotted in (c)-(d).

reconstructed fields decrease, as do the ones relative to the missing data (converged Nash strategies, which we recall are, respectively, the trace of $u_{1}$ and the normal trace of $u_{2}$ over the sphere $\Gamma_{i}$ ).

The sensitivity of the reconstructed fields and missing data to the noise level $\sigma$ is shown in Figure 5.7. Interestingly, boundary missing data are much less sensitive than the domain distributed fields. Both exhibit a satisfactory stable behavior w.r.t. the noise magnitude.

Test case $\mathbf{D}$. The second function is given by

$$
u(x, y, z)=\frac{1}{\sqrt{(x+0.2)^{2}+y^{2}+z^{2}}} .
$$

The function $u$ given by (5.5) is the nonradial solution of $\Delta u=\delta_{X_{0}}$, where the source term is now $X_{0}=(-0.2,0,0)$.

In this experiment we put $5 \%$ of noise in $(f, \Phi)$ according to (5.1). The obtained results are illustrated in Figure 5.8, where the reconstructed fields $u_{1}$ and $u_{2}$ are presented at convergence. The nonradial missing data are presented in Figure 5.9 


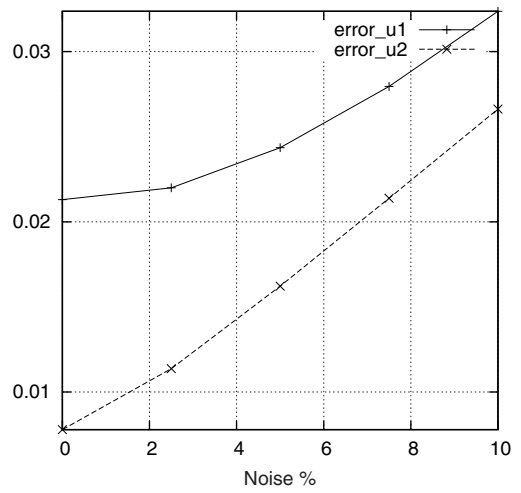

(a)

Fig. 5.7. Test case C. Sensitivity of the reconstructed fields to noisy Cauchy data $\left(f^{\sigma}, \Phi^{\sigma}\right)$. $L^{2}$-errors are presented as a function of the noise level $\sigma$ : (a) reconstructed fields: $\left\|u_{i}-u\right\| /\|u\|$, $i=1,2$; (b) missing data: Dirichlet $\left\|\tau_{N}-u_{\mid \Gamma_{i}}\right\| /\left\|u_{\mid \Gamma_{i}}\right\|$ and Neumann $\left\|\eta_{N}-\frac{\partial u}{\partial \nu} \mid \Gamma_{i}\right\| /\left\|\frac{\partial u}{\partial \nu}_{\mid \Gamma_{i}}\right\|$.

(a)

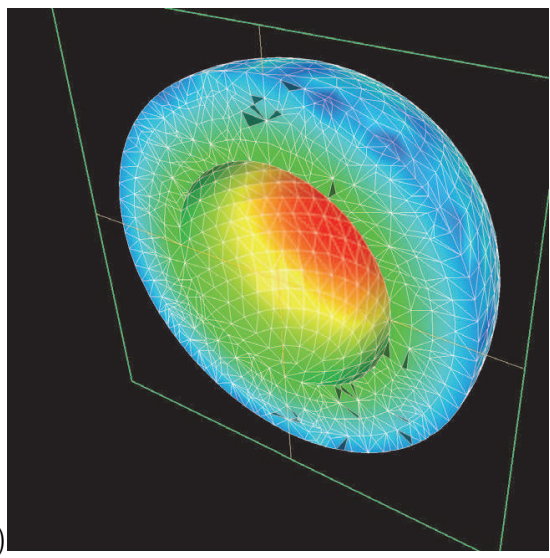

(b)

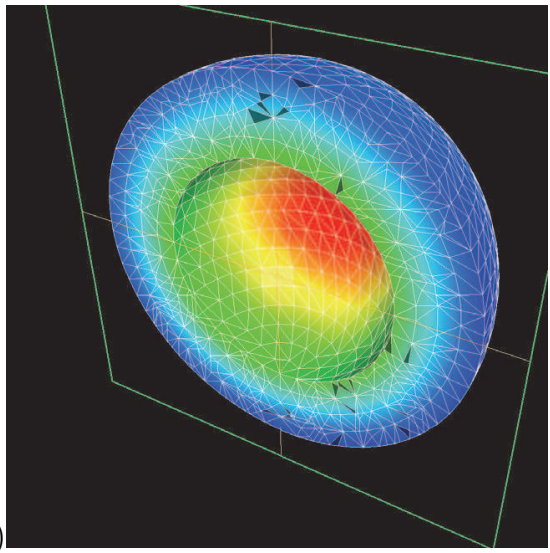

Fig. 5.8. Test case D. Nonradial case. Noise level is 5\%. The level sets of the reconstructed fields (a) $u_{1}$ and (b) $u_{2}$ are presented at convergence. The finite element computations are performed with 4740 nodes and 22,795 tetrahedral elements.

and relative $L^{2}$-errors on the reconstructed fields and boundary data are shown in Figure 5.10.

Even though the error curves do monotonically decrease toward zero for the two test cases $\mathrm{C}$ and $\mathrm{D}$, the stagnation that appears quite early (around iterations 120-160 in the present case) suggests that multilevel or hierarchical optimization approaches should be designed to speed up the convergence of the Nash algorithm.

In Figure 5.11, we compare for the 3D test case D the sensitivities of the controltype method versus the Nash game algorithm with respect to the noise level $\sigma$. The $L^{2}$-error on Dirichlet and Neumann missing data are plotted, confirming the advantage of the game algorithm already observed in the $2 \mathrm{D}$ comparison case. The difference between the two reconstruction methods is much more striking as the noise level increases. This advantage of the Nash game approach, however, should be moderated by the observation that the overall Nash do loop needs to solve slightly more optimization subtasks than the control-type methods. Parallel implementations should help to overcome this drawback. 

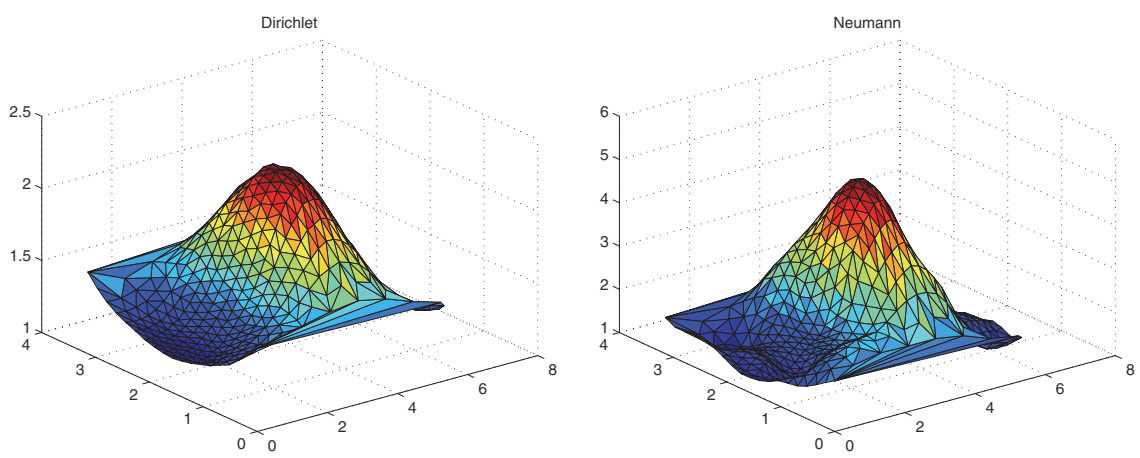

Fig. 5.9. Test case D. Reconstructed nonradial Dirichlet $\left(\tau_{N}\right.$, left) and Neumann ( $\eta_{N}$, right) data over $\Gamma_{i}$. The profiles are presented at convergence and for a noise level $\sigma=5 \%$.

(a)

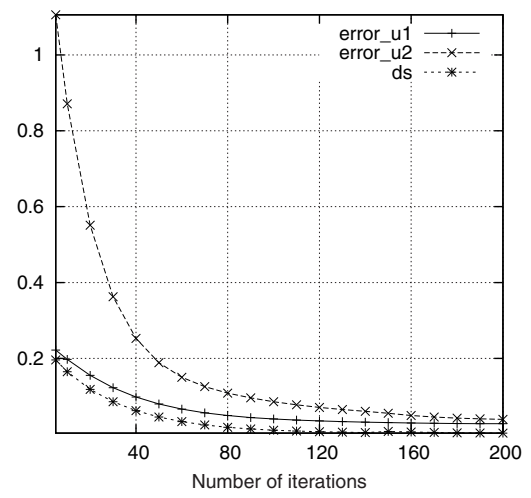

(b)

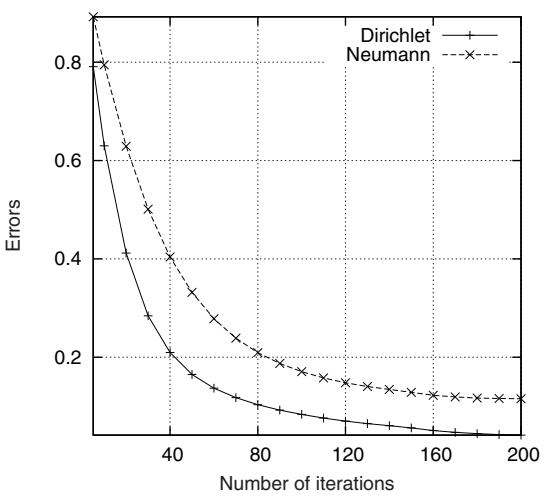

FIG. 5.10. Test case D. Relative $L^{2}$-errors are presented for the nonradial case as a function of the overall Nash iterations. Noise level is 5\%. (a) reconstructed fields error: $\left\|u_{i}^{(k)}-u\right\| /\|u\|$, $i=1,2$ and Nash strategies $d s=\left\|S^{(k)}-S^{(k-1)}\right\|$; (b) missing Dirichlet data: $\left\|\tau^{(k)}-u_{\mid \Gamma_{i}}\right\| /\left\|u_{\mid \Gamma_{i}}\right\|$ and Neumann data $\left\|\eta^{(k)}-\frac{\partial u}{\partial \nu}{ }_{\mid \Gamma_{i}}\right\| /\left\|\left.\frac{\partial u}{\partial \nu}\right|_{\Gamma_{i}}\right\|$.
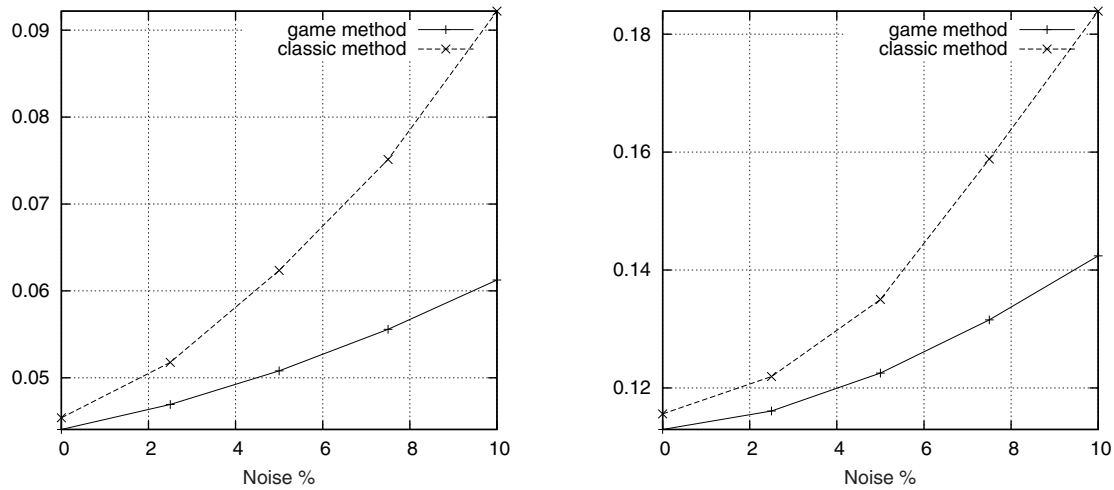

FIG. 5.11. Test case D. Robustness of the control-type (classic) method compared to the Nash (game) method. Sensitivity of the corresponding reconstructed missing data to noisy Cauchy data $\left(f^{\sigma}, \Phi^{\sigma}\right) . L^{2}$-errors are presented as a function of the noise level $\sigma:$ (left) Dirichlet $\left\|\tau_{N}-u_{\mid \Gamma_{i}}\right\| /$ $\left\|u_{\mid \Gamma_{i}}\right\|$ and (right) Neumann $\left\|\eta_{N}-\frac{\partial u}{\partial \nu}{ }_{\mid \Gamma_{i}}\right\| /\left\|\frac{\partial u}{\partial \nu} \mid \Gamma_{i}\right\|$.

Copyright $@$ by SIAM. Unauthorized reproduction of this article is prohibited. 
6. Conclusion. Let us conclude the paper with some short remarks. First, we have used the simplest class of games to model the completion problem, namely, the class of static games with complete information. This simple game formulation yields interesting results like the existence and uniqueness of a Nash equilibrium, which is exactly the missing data when the Cauchy problem has a solution. The decomposition not only of the control variable but also of the criteria allows for standard and simple mathematical analysis to be used to prove stability results with respect to perturbation of the Cauchy data. Investigation of more sophisticated classes of games such as dynamical games with incomplete information may lead to new efficient data completion algorithms.

Our method may be extended as it is to the linear elasticity and to the Stokes systems; see [2] where a control-type setting for Stokes system has been studied. Existence, uniqueness of a Nash equilibrium, and stability properties should hold with minimal adaption of the proofs. An application of the game algorithm to a nonlinear elliptic case (Perona-Malik model for image inpainting) is ongoing.

It is also interesting to notice that solving the data completion problem with our method makes use of the standard computational tools, be it finite element or optimization codes. The numerical experiments presented for different 2D and 3D test cases tend to prove that our method is efficient and exhibits better numerical stability with respect to noisy Cauchy data than the classical control-type approaches.

\section{REFERENCES}

[1] R. Aboulä̈ch, A. Ben Abda, And M. Kallel, Missing boundary data reconstruction via an approximate optimal control, Inverse Problems Imaging, 2 (2008) pp. 411-426.

[2] R. Aboulä̈ch, A. Ben Abda and M. Kallel, A control type method for solving the CauchyStokes problem, Appl. Math. Model., 37 (2013), pp. 4295-4304.

[3] G. Alessandrini, L. Rondi, E. Rosset, and S. Vessella, The stability for the Cauchy problem for elliptic equations, Inverse Problems, 25 (2009), 123004.

[4] S. Andrieux, T. Baranger, And A. Ben Abda, Solving Cauchy problems by minimizing an energy-like functional, Inverse Problems, 22 (2006) pp. 115-133.

[5] H. Attouch, P. Redont, and A. Soubeyran, A new class of alternating proximal minimization algorithms with costs-to-move, SIAM J. Optim., 18 (2007), pp. 1061-1081.

[6] H. Attouch, J. Bolte, P. Redont, and A. Soubeyran, Alternating proximal algorithms for weakly coupled convex minimization problems. Applications to dynamical games and PDE's, J. Convex Anal., 15 (2008), pp. 485-506.

[7] H. Attouch And M. Soueycatt, Augmented Lagrangian and proximal alternating direction methods of multipliers in Hilbert spaces. Applications to games, PDE's and control, Pacific J. Optim, 5 (2009), pp. 17-37.

[8] T. BASAR, Relaxation techniques and asynchronous algorithms for on-line computation of noncooperative equilibria, J. Econom. Dynam. Control, 11 (1987), pp. 531-549.

[9] A. Ben Abda, M. Kallel, J. Leblond, and J.-P. Marmorat, Line-segment cracks recovery from incomplete boundary data, Inverse Problems, 18 (2002), pp. 1057-1077.

[10] A. Ben Abda, F. Ben Hassen, J. Leblond, and M. Mahjoub, Sources recovery from boundary data: A model related to electroencephalography, Math. Comput. Modelling, 49 (2009), pp. $2213-2223$.

[11] F. Ben Belgacem, Why is the Cauchy problem severely ill-posed?, Inverse Problems, 23 (2007), pp. 823-836.

[12] L. Bourgeois, About stability and regularization of ill-posed elliptic Cauchy problems: The case of $C^{1,1}$ domains, M2AN Math. Model. Numer. Anal., 44 (2010), pp. 715-735.

[13] S. ChaAbane, M. Jaoua, And J. Leblond, Parameter identification for Laplace equation and approximation in analytic classes, J. Inverse Ill-Posed Probl., 11 (2003), pp. 35-57.

[14] H. CaO AND S. V. Pereverzv, The balancing principle for the regularization of elliptic Cauchy problems, Inverse Problems, 23 (2007), pp. 1943-1961.

[15] A. ChakiB And A. Nachaoui, Convergence analysis for finite element approximation to an inverse Cauchy problem, Inverse Problems 22 (2006), pp. 1191-1206. 
[16] A. Cimetière, F. Delvare, M. Jaoua, and F. Pons, Solution of the Cauchy problem using iterated Tikhonov regularization, Inverse Problems, 17 (2001), pp. 553-570.

[17] A. Cimetière, F. Delvare, M. Jaoua, M. Kallel, and F. Pons, Recovery of cracks from incomplete boundary data, Inverse Problems Engrg., 10 (2002), pp. 377-392.

[18] H. Dinh Nho and D. Lesnic, The Cauchy problem for Laplace's equation via the conjugate gradient method, IMA J. Appl. Math., 65 (2000), pp. 199-217.

[19] A. A. Habbal, Topology Nash game for tumoral antiangiogenesis, Struct. Multidisciplinary Optim., 30 (2005), pp. 404-412.

[20] A. Habbal, J. Petersson, and M. Thellner, Multidisciplinary topology optimization solved as a Nash game, Internat. J. Numer. Methods Engrg., 61 (2004), pp. 949-963.

[21] J. Hadamard, Lectures on Cauchy's Problem in Linear Partial Differential Equation, Dover, New York, 1953.

[22] F. Hecht, A. Le Hyaric, and O. Pironneau, Freefem ++ , http://www.freefem.org/ff++/.

[23] V. A. Kozlov, V. G. Maz'ya, And A. V. Fomin, An iterative method for solving the Cauchy problems for elliptic equations, Comput. Math. Phys., 31 (1991), pp. 45-52.

[24] S. Li AND T. BASAR, Distributed algorithms for the computation of noncooperative equilibria, Automatica 23 (1987), pp. 523-533.

[25] A. Borzì AND C. KANzow, Formulation and numerical solution of Nash equilibrium multiobjective elliptic control problems, SIAM J. Control Optim., 51 (2013), pp. 718-744.

[26] J.-A. DesiderI, Cooperation and competition in multidisciplinary optimization. Application to the aero-structural aircraft wing shape optimization, Comput. Optim. Appl., 52 (2012), pp. 29-68.

[27] A. Quarteroni And A. Valli, Theory and application of Steklov-Poincare operators for boundary value problems, in Applied and Industrial Mathematics, R. Spigler, ed., Kluwer, Dordrecht, 1991, pp. 179-203.

[28] S. URYAS'EV AND R. Y. Rubinstein, On relaxation algorithms in computation of noncooperative equilibria, IEEE Trans. Automat. Control, 39 (1994), pp. 1263-1267.

Copyright $@$ by SIAM. Unauthorized reproduction of this article is prohibited. 